\title{
Fixed point Theorems of Multivalued Mappings in Cone Metric Spaces via Cone $\boldsymbol{C}$-Class function
}

\author{
R. Krishnakumar ${ }^{1}$, K. Dinesh ${ }^{2 *}$, Arslan Hojat Ansari ${ }^{3}$ \\ ${ }^{1}$ PG \& Research Department of Mathematics,Urumu Dhanalakshmi College, Tiruchirappalli-19, Tamilnadu, India \\ ${ }^{2}$ PG \& Research Department of Mathematics,Urumu Dhanalakshmi College,Tiruchirappalli-19, Tamilnadu, India \\ ${ }^{3}$ Department of Mathematics, Karaj Branch, Islamic Azad University, Karaj, Iran. \\ *Corresponding Author:dinesh.skksv93@gmail.com, Tel.: +917418865975
}

Available online at: www.isroset.org

Accepted 18/Aug/2018, Online 30/Aug/2018

$\boldsymbol{A b s t r a c t - L e t ~} \boldsymbol{P}$ be a subset of a Banach space $\boldsymbol{E}$ and $\boldsymbol{P}$ is normal and regular cone on $\boldsymbol{E}$, we prove the existence of the fixed point for multi valued maps and $\varphi-\psi$ - contractive mappings in cone metric spaces via cone $\mathrm{C}$ class functions.

Keywords - Cone metric space, Multivalued mappings, Fixed point, Cone C class function

\section{INTRODUCTION}

In recent years, several authors (see [1-5]) have studied the strong convergence to a fixed point with contractive constant in cone metric spaces. Seong Hoon Cho and Mi sun Kim [5] have proved certain fixed point theorems by using Multivalued mapping in the setting of contractive constant in metric spaces. Note on $\varphi-\psi$-cotractive type mappings and related fixed point are proved by Arslan Hojat Ansari [8]. Fixed point theorems of Multivalued mappings in Cone metric spaces proved by Dr.M.Marudai and Dr.R.Krishnakumar [1].

\section{PRELIMINARIES}

Definition 1.1: Let $E$ be a Banach space and a subset, $P$ of $E$ is said to be a cone if it satisfies the following conditions (i) $P \neq \emptyset$ and $P$ is closed;

(ii) $a x+b y \in P \forall x, y \in P$ and $a, b$ are non-negative real numbers

(iii) $P \cap(-P)=\emptyset$

Given a cone $P \subset E$,we define a partial ordering $\leq$ with respect to the cone $P$ by $x \leq y$ if and only if $y-x \in P$. If $y-x \in$ interior of $P$, then it is denoted by $x \ll y$. The cone $P$ is said to be Normal if a number $K>0$ such that for all $x, y \in E$, $0 \leq x \leq y$ implies $\|x\| \leq K\|y\|$. The cone $P$ is called regular if every increasing sequence which is bounded above is convergent and every decreasing sequence which is bounded below convergent.

Definition 1.2 : Let $X$ be a non-empty set, and suppose the mapping $d: X \times X \rightarrow E$ is said to be a cone metric space if it satisfies

(i) $0 \leq d(x, y) \forall x, y \in X$ and $d(x, y)=0$ if and only if $x=y$

(ii) $d(x, y)=d(y, x)$ for all $x, y \in X$

(iii) $d(x, y)=d(x, z)+d(z, y)$ for all $x, y, z \in X$

Example 1.3: Let $E=R^{2}, P=\{(x, y) \in E ; x, y \geq 0\}, X=R$ and $d: X \times X \rightarrow E$ defined by

Where $\alpha \geq 0$ is a constant. Then $(X, d)$ is a cone metric space.

$$
d(x, y)=(|x-y|, \alpha|x-y|)
$$

Definition 1.4: Let $(X, d)$ be cone metric space, $x \in X$ and $\left\{x_{n}\right\}$ a sequence in $X$. Then

(i) $\quad\left\{x_{n}\right\}$ converges to $x$ whenever for every $c \in E$ with $0 \ll c$ there is a natural number $N$ such that $d\left(x_{n}, x\right) \ll c$ for all $n \geq N$ 
(ii) $\quad\left\{x_{n}\right\}$ is a Cauchy sequence whenever for every $c \in E$ with $0 \ll c$ there is a natural number $N$ such that $d\left(x_{n}, x_{m}\right) \ll c$ for all $n, m \geq N$.

Definition 1.5. Let $(X, d)$ is said to be a complete cone metric space, if every Cauchy sequence is convergent in $X$

Definition1.6: Let $(X, d)$ be a metric space. We denote $C B(X)$ the family of nonempty closed bounded subset of $X$. Let $H(.,$. be the Hausdorff distance on $C B(X)$.

That is, for $A, B \in C B(X)$

$H(A, B)=\max \left\{\sup _{\mathrm{a} \in \mathrm{A}} d(a, B), \sup _{\mathrm{b} \in \mathrm{B}} d(A, b)\right\}$

Where $d(a, B)=\inf \{d(a, b) ; b \in B\}$ is the distance from the point $a$ to the subset $B$. An element $x \in X$ is said to be a fixed point of a multi-valued mapping $T: X \rightarrow 2^{X}$ if $x \in T(X)$

Definition 1.7: A function $\psi: P \rightarrow P$ is called an altering distance function if the following properties are satisfied:

(i) $\psi$ is non-decreasing and continuous

(ii) $\psi(t)=0$ if and only if $t=0$

Definition 1.8. : An ultra altering distance function is a continuous, non decreasing mapping $\varphi: P \rightarrow P$ such that $\varphi(t)>0$, $t>0$ and $\varphi(0) \geq 0$

We denote this set with $\Phi_{u}$

Definition 1.9.: A mapping $f: P^{2} \rightarrow P$ is called cone $C$-class function if it is continuous and satisfies following axioms:

(1) $F(s, t)<s$;

(2) $F(s, t)=s$ implies that either $s=0$ or $t=0$; for all $s, t \in P$

We denote cone $C$-class functions as $\mathcal{C}$

Example 2.9 : The following functions $F: P^{2} \rightarrow P$ are elements of $\mathcal{C}$, for all $s, t \in[0, \infty)$ :

(i) $F(s, t)=s-t$

(ii) $F(s, t)=k s$, where $0<k<1$,

(iii) $F(s, t)=s \beta(s)$, where $\beta:[0, \infty) \rightarrow[0,1)$,

(iv) $\quad F(s, t)=\Psi(s)$, where $\Psi: \mathrm{P} \rightarrow \mathrm{P}, \Psi(0)=0, \Psi(\mathrm{s})>0$ for all $s \in P$ with $s \neq 0$ and $\Psi(s) \leq s$ for all $s \in P$

(v) $\quad F(s, t)=s-\varphi(s)$, where $\varphi:[0, \infty) \rightarrow[0, \infty)$ is a continuous function such that $\varphi(t)=0 \Leftrightarrow t=0$;

(vi) $\quad F(s, t)=s-h(s, t)$, where $h:[0, \infty) \times[0, \infty) \rightarrow[0, \infty)$ is a continuous function such that $h(s, t)=0 \Leftrightarrow t=0$ for all $t, s>0$

(vii) $F(s, t)=\varphi(s), F(s, t)=s \Rightarrow s=0$, here $\varphi:[0, \infty) \rightarrow[0, \infty)$ is a upper semi continuous function such that $\varphi(0)=0$ and $\varphi(t)<t$ for $t>0$

Lemma 1.10: Let $\psi$ and $\varphi$ are altering distance and ultra altering distance functions respectively, $F \in \mathcal{C}$ and $\left\{s_{n}\right\}$ a decreasing sequence in $P$ such that

For all $n \geq 1$. Then $\lim _{\mathrm{n} \rightarrow \infty} s_{n}=0$

$$
\psi\left(s_{n+1}\right) \leq F\left(\psi\left(s_{n}\right), \varphi\left(s_{n}\right)\right)
$$

\section{MAIN RESULTS}

Theorem 2.1: Let $(X, d)$ be a complete cone metric space and the mapping $T: X \rightarrow C B(X)$ be multivalued map satisfying for each $x, y \in X$

$$
\begin{aligned}
& \psi(H(T x, T y)) \leq F(\psi[a[d(x, T x)+d(y, T y)]+b[d(x, T y)+d(T x, y)]] \\
& \qquad \phi(a[d(x, T x)+d(y, T y)]+b[d(x, T y)+d(T x, y)]) \text { for all } x, y \in X \text { and } a+b<\frac{1}{2}, a, b \in\left[0, \frac{1}{2}\right) \cdot \psi \text { and } \\
& \varphi \text { are altering distance and ultra altering distance functions respectively, } F \in \mathcal{C} \text { such that } \psi(t+s) \leq \psi(t)+\psi(s) \text {. Then } T \\
& \text { has a fixed point in } X \\
& \text { Proof: for every } x_{0} \in X \text { and } n \geq 1, x_{1} \in T x_{0} \text { and } x_{n+1} \in T x_{n} \\
& \psi\left(d\left(x_{n+1}, x_{n}\right)\right) \leq \psi\left(H\left(T x_{n}, T x_{n-1}\right)\right) \\
& \quad \leq F\left(\psi\left(a\left[d\left(x_{n}, T x_{n}\right)+d\left(x_{n-1}, T x_{n-1}\right)\right]+b\left[d\left(x_{n}, T x_{n-1}\right)+d\left(T x_{n}, x_{n-1}\right)\right]\right)\right.
\end{aligned}
$$

$\varphi$ are altering distance and ultra altering distance functions respectively, $F \in \mathcal{C}$ such that $\psi(t+s) \leq \psi(t)+\psi(s)$. Then $T$ 


$$
\begin{aligned}
& \left.\quad \phi\left(a\left[d\left(x_{n}, T x_{n}\right)+d\left(x_{n-1}, T x_{n-1}\right)\right]+b\left[d\left(x_{n}, T x_{n-1}\right)+d\left(T x_{n}, x_{n-1}\right)\right]\right)\right) \\
& \leq F\left(\psi\left(a\left[d\left(x_{n}, x_{n+1}\right)+d\left(x_{n-1}, x_{n}\right)\right]+b\left[d\left(x_{n}, x_{n}\right)+d\left(x_{n+1}, x_{n-1}\right)\right]\right),\right. \\
& \left.\varphi\left(a\left[d\left(x_{n}, x_{n+1}\right)+d\left(x_{n-1}, x_{n}\right)\right]+b\left[d\left(x_{n}, x_{n}\right)+d\left(x_{n+1}, x_{n-1}\right)\right]\right)\right) \\
& \leq F\left(\psi\left(a\left[d\left(x_{n}, x_{n+1}\right)+d\left(x_{n-1}, x_{n}\right)\right]+b\left[d\left(x_{n+1}, x_{n-1}\right)\right]\right),\right. \\
& \left.\quad \varphi\left(a\left[d\left(x_{n}, x_{n+1}\right)+d\left(x_{n-1}, x_{n}\right)\right]+b\left[d\left(x_{n+1}, x_{n-1}\right)\right]\right)\right) \\
& \leq F\left(\psi\left(a\left[d\left(x_{n}, x_{n+1}\right)+d\left(x_{n-1}, x_{n}\right)\right]+b\left[d\left(x_{n+1}, x_{n}\right)+d\left(x_{n}, x_{n-1}\right)\right]\right),\right. \\
& \left.\quad \varphi\left(a\left[d\left(x_{n}, x_{n+1}\right)+d\left(x_{n-1}, x_{n}\right)\right]+b\left[d\left(x_{n+1}, x_{n}\right)+d\left(x_{n}, x_{n-1}\right)\right]\right)\right) \\
& \leq F\left(\psi\left((a+b)\left[d\left(x_{n}, x_{n+1}\right)+d\left(x_{n-1}, x_{n}\right)\right]\right), \varphi\left((a+b)\left[d\left(x_{n}, x_{n+1}\right)+d\left(x_{n-1}, x_{n}\right)\right]\right)\right) \\
& \leq \psi\left((a+b)\left[d\left(x_{n}, x_{n+1}\right)+d\left(x_{n-1}, x_{n}\right)\right]\right) \\
& \begin{array}{l}
d\left(x_{n+1}, x_{n}\right) \leq L d\left(x_{n-1}, x_{n}\right) \quad \text { where } L=\frac{a+b}{1-(a+b)} \\
d\left(x_{n+1}, x_{n}\right) \leq L^{n} d\left(x_{1}, x_{0}\right) \\
\text { For } n>m \text { we have } \\
d\left(x_{n}, x_{m}\right) \leq d\left(x_{n}, x_{n-1}\right)+d\left(x_{n-1}, x_{n-2}\right)+\cdots+d\left(x_{m+1}, x_{m}\right) \\
\leq\left[L^{n-1}+L^{n-2}+\cdots+L^{m}\right] d\left(x_{1}, x_{0}\right) \\
\leq \frac{L^{m}}{(1-L)} d\left(x_{1}, x_{0}\right)
\end{array}
\end{aligned}
$$

Let $0 \ll c$ be given, choose a natural number $N_{1}$ such that $\frac{L^{m}}{(1-L)} d\left(x_{1}, x_{0}\right) \ll c$ for all $m \geq N_{1}$ this implies $d\left(x_{n}, x_{m}\right) \ll c$. For $n>m,\left\{x_{n}\right\}$ is a Cauchy sequence in $(X, d)$ is a complete cone metric space, there exists $p \in X$ such that $x_{n} \rightarrow p$. Choose a natural number $N_{2}$ such that $d\left(x_{n}, P\right) \ll \frac{c(1-L)}{3}$, for all $n \geq N_{2}$. Hence for $n \geq N_{2}$ we have $d\left(x_{n}, P\right) \ll \frac{c(1-K)}{3}$ where

$$
\begin{aligned}
& k=a+b \\
& \psi(d(T p, P)) \leq \psi\left(H\left(T x_{n}, T p\right)+d\left(T x_{n}, p\right)\right) \\
& \leq F\left(\psi\left(a\left[d\left(x_{n}, T x_{n}\right)+d(p, T p)\right]+b\left[d\left(x_{n}, T p\right)+d\left(T x_{n}, p\right)\right]+d\left(x_{n+1}, p\right)\right),\right. \\
& \left.\varphi\left(a\left[d\left(x_{n}, T x_{n}\right)+d(p, T p)\right]+b\left[d\left(x_{n}, T p\right)+d\left(T x_{n}, p\right)\right]+d\left(x_{n+1}, p\right)\right)\right) \\
& \leq F\left(\psi\left(a\left[d\left(x_{n}, x_{n+1}\right)+d(p, T p)\right]+b\left[d\left(x_{n}, T p\right)+d\left(x_{n+1}, p\right)\right]+d\left(x_{n+1}, p\right)\right)\right. \\
& , \varphi\left(a\left[d\left(x_{n}, x_{n+1}\right)+d(p, T p)\right]+b\left[d\left(x_{n}, T p\right)+d\left(x_{n+1}, p\right)\right]+d\left(x_{n+1}, p\right)\right) \\
& \leq F\left(\psi\left(a\left[d\left(x_{n}, x_{n+1}\right)+d(p, T p)\right]+b\left[d\left(x_{n}, T p\right)+d(p, T p)+d\left(x_{n+1}, p\right)\right]+d\left(x_{n+1}, p\right)\right),\right. \\
& \varphi\left(a\left[d\left(x_{n}, x_{n+1}\right)+d(p, T p)\right]+b\left[d\left(x_{n}, T p\right)+d(p, T p)+d\left(x_{n+1}, p\right)\right]+d\left(x_{n+1}, p\right)\right) \\
& \leq F\left(\psi\left(a d\left(x_{n}, x_{n+1}\right)+a d(p, T p)+b d\left(x_{n}, T p\right)+b d(p, T p)+b d\left(x_{n+1}, p\right)+d\left(x_{n+1}, p\right)\right)\right. \text {, } \\
& \left.\varphi\left(\operatorname{ad}\left(x_{n}, x_{n+1}\right)+a d(p, T p)+b d\left(x_{n}, T p\right)+b d(p, T p)+b d\left(x_{n+1}, p\right)+d\left(x_{n+1}, p\right)\right)\right) \\
& \leq\left(a d\left(x_{n}, x_{n+1}\right)+a d(p, T p)+b d\left(x_{n}, T p\right)+b d(p, T p)+b d\left(x_{n+1}, p\right)+d\left(x_{n+1}, p\right)\right. \\
& (1-k) d(T p, p) \leq k d\left(x_{n}, T p\right)+k d\left(x_{n+1}, p\right)+d\left(x_{n+1}, p\right) \\
& \leq d\left(x_{n}, T p\right)+d\left(x_{n+1}, p\right)+d\left(x_{n+1}, p\right) \\
& d(T p, p) \leq \frac{\left[d\left(x_{n}, T p\right)+d\left(x_{n+1}, p\right)+d\left(x_{n+1}, p\right)\right]}{(1-k)} \\
& d(T p, p) \ll \frac{c}{3}+\frac{c}{3}+\frac{c}{3} \\
& d(T p, p) \ll c
\end{aligned}
$$

For all $n \geq N_{2}, d(T p, p) \ll \frac{c}{m}$ for all $m \geq 1$, we get $\frac{c}{m}-d(T p, p) \in P$ and $m \rightarrow \infty$ we get $\frac{c}{m} \rightarrow 0$ and $P$ is closed $d(T p, p) \in$ $P$ bu $d(T p, p) \in P$

$\therefore d(T p, p)=0$ and so $p \in T p$.

Corollary 2.1: Let $(X, d)$ be a complete cone metric space and the mapping $T: X \rightarrow C B(X)$ be multivalued map satisfying for each $x, y \in X$

$$
\psi(d(T x, T y)) \leq F(\psi(a[d(x, T x)+d(y, T y)]), \varphi(a[d(x, T x)+d(y, T y)]))
$$

for all $x, y \in X$ and $a \in\left[0, \frac{1}{2}\right) . \psi$ and $\varphi$ are altering distance and ultra altering distance functions respectively, $F \in \mathcal{C}$ such that $\psi(t+s) \leq \psi(t)+\psi(s)$. Then $T$ has a fixed point in $X$

Proof: The proof of the corollary immediately follows by putting $b=0$ in the previous theorem.

Theorem 2.2: Let $(X, d)$ be a complete cone metric space and the mapping $T: X \rightarrow C B(X)$ be multivalued map satisfy the condition

$$
\psi(H(T x, T y)) \leq F(\psi(r \max \{d(x, y), d(x, T x), d(y, T y)\}), \varphi(r \max \{d(x, y), d(x, T x), d(y, T y)\}))
$$


For all $x, y \in X$ and $r \in[0,1) . \psi$ and $\varphi$ are altering distance and ultra altering distance functions respectively, $F \in \mathcal{C}$ such that $\psi(t+s) \leq \psi(t)+\psi(s)$. Then $T$ has a fixed point in $X$

Proof: for every $x_{0} \in X$ and $n \geq 1, x_{1} \in T x_{0}$ and $x_{n+1} \in T x_{n}$

$\psi\left(d\left(x_{n+1}, x_{n}\right)\right) \leq \psi\left(H\left(T x_{n}, T x_{n-1}\right)\right)$

$\leq F\left(\psi\left(r \max \left\{d\left(x_{n}, x_{n-1}\right), d\left(x_{n}, T x_{n}\right), d\left(x_{n-1}, T x_{n-1}\right)\right\}\right), \varphi\left(r \max \left\{d\left(x_{n}, x_{n-1}\right), d\left(x_{n}, T x_{n}\right), d\left(x_{n-1}, T x_{n-1}\right)\right\}\right)\right)$

$\leq F\left(\psi\left(r \max \left\{d\left(x_{n}, x_{n-1}\right), d\left(x_{n}, x_{n+1}\right), d\left(x_{n-1}, x_{n}\right)\right\}\right), \varphi\left(r \max \left\{d\left(x_{n}, x_{n-1}\right), d\left(x_{n}, x_{n+1}\right), d\left(x_{n-1}, x_{n}\right)\right\}\right)\right)$

$\leq F\left(\psi\left(r d\left(x_{n}, x_{n-1}\right)\right), \varphi\left(r d\left(x_{n}, x_{n-1}\right)\right)\right)$

$\leq r^{n} d\left(x_{1}, x_{0}\right)$

For $n>m$ we have

$$
\begin{aligned}
d\left(x_{n}, x_{m}\right) & \leq d\left(x_{n}, x_{n-1}\right)+d\left(x_{n-1}, x_{n-2}\right)+\cdots+d\left(x_{m+1}, x_{m}\right) \\
& \leq\left[r^{n-1}+r^{n-2}+\cdots+r^{m}\right] d\left(x_{1}, x_{0}\right) \\
& \leq \frac{r^{m}}{(1-r)} d\left(x_{1}, x_{0}\right)
\end{aligned}
$$

Let $0 \ll c$ be given, choose a natural number $N_{1}$ such that $\frac{r^{m}}{(1-r)} d\left(x_{1}, x_{0}\right) \ll c$ for all $m \geq N_{1}$ this implies $d\left(x_{n}, x_{m}\right) \ll c$. For $n>m,\left\{x_{n}\right\}$ is a Cauchy sequence in $(X, d)$ is a complete cone metric space, there exists $p \in X$ such that $x_{n} \rightarrow p$. Choose a natural number $N_{2}$ such that $d\left(x_{n}, P\right) \ll \frac{c}{3}$, for all $n \geq N_{2}$. Hence for $n \geq N_{2}$ we have $d\left(x_{n}, P\right) \ll \frac{c}{3}$

$$
\begin{aligned}
\psi(d(T p, P)) \leq & \psi\left(H\left(T x_{n}, T p\right)+d\left(T x_{n}, p\right)\right) \\
\leq & F\left(\psi\left(r \max \left\{d\left(x_{n}, p\right), d\left(x_{n}, T x_{n}\right), d(p, T p)\right\}+d\left(x_{n+1}, p\right)\right),\right. \\
& \left.\varphi\left(r \max \left\{d\left(x_{n}, p\right), d\left(x_{n}, T x_{n}\right), d(p, T p)\right\}+d\left(x_{n+1}, p\right)\right)\right) \\
\leq & F\left(\psi\left(r \max \left\{d\left(x_{n}, p\right), d\left(x_{n}, x_{n+1}\right), d(p, T p)\right\}+d\left(x_{n+1}, p\right)\right),\right. \\
& \left.\varphi\left(r \max \left\{d\left(x_{n}, p\right), d\left(x_{n}, x_{n+1}\right), d(p, T p)\right\}+d\left(x_{n+1}, p\right)\right)\right) \\
\leq & F\left(\psi\left(r \max \left\{d\left(x_{n}, p\right), d\left(x_{n}, p\right)+d\left(p, x_{n+1}\right), d(p, T p)\right\}+d\left(x_{n+1}, p\right)\right),\right. \\
& \varphi\left(r \max \left\{d\left(x_{n}, p\right), d\left(x_{n}, p\right)+d\left(p, x_{n+1}\right), d(p, T p)\right\}+d\left(x_{n+1}, p\right)\right)
\end{aligned}
$$

$d(T p, P) \ll c$

For all $n \geq N_{2}, d(T p, p) \ll \frac{c}{m}$ for all $m \geq 1$, we get $\frac{c}{m}-d(T p, p) \in P$ and $m \rightarrow \infty$ we get $\frac{c}{m} \rightarrow 0$ and $P$ is closed $-d(T p, p) \in$ $P$ bu $d(T p, p) \in P$

$\therefore d(T p, p)=0$ and so $p \in T p$.

Corollary 2.2: Let $(X, d)$ be a complete cone metric space and the mapping $T: X \rightarrow C B(X)$ be multivalued map satisfy the condition

$$
H(T x, T y) \leq k d(x, y)
$$

For all $x, y \in X$ and $k \in[0,1) . \psi$ and $\varphi$ are altering distance and ultra altering distance functions respectively, $F \in \mathcal{C}$ such that $\psi(t+s) \leq \psi(t)+\psi(s)$. Then $T$ has a fixed point in $X$

Proof: The proof of the corollary immediately follows by taking $d(x, y)$ as maximum value in previous theorem.

Note 2.3: We prove the above theorems in the setting of $P$ is a normal cone with normal constant $K$

Theorem 2.4: Let $(X, d)$ be a complete cone metric space and $P$ a normal cone with normal constant $K$. Suppose the mapping $T: X \rightarrow C B(X)$ be multivalued map satisfy the condition

$\psi(H(T x, T y)) \leq F(\psi(r \max \{d(x, y), d(x, T x), d(y, T y), d(x, T y), d(T x, y)\})$,

$$
\varphi(r \max \{d(x, y), d(x, T x), d(y, T y), d(x, T y), d(T x, y)\}))
$$

For all $x, y \in X$ and $r \in[0,1) . \psi$ and $\varphi$ are altering distance and ultra altering distance functions respectively, $F \in \mathcal{C}$ such that $\psi(t+s) \leq \psi(t)+\psi(s)$. Then $T$ has a fixed point in $X$

Proof: for every $x_{0} \in X$ and $n \geq 1, x_{1} \in T x_{0}$ and $x_{n+1} \in T x_{n}$

$$
\begin{aligned}
& \psi\left(d\left(x_{n+1}, x_{n}\right)\right) \leq \psi\left(H\left(T x_{n}, T x_{n-1}\right)\right) \\
& \leq F\left(\psi\left(r \max \left\{d\left(x_{n}, x_{n-1}\right), d\left(x_{n}, T x_{n}\right), d\left(x_{n-1}, T x_{n-1}\right), d\left(x_{n}, T x_{n-1}\right), d\left(T x_{n}, x_{n-1}\right)\right\}\right),\right. \\
&\left.\varphi\left(r \max \left\{d\left(x_{n}, x_{n-1}\right), d\left(x_{n}, T x_{n}\right), d\left(x_{n-1}, T x_{n-1}\right), d\left(x_{n}, T x_{n-1}\right), d\left(T x_{n}, x_{n-1}\right)\right\}\right)\right) \\
& \leq F\left(\psi\left(r \max \left\{d\left(x_{n}, x_{n-1}\right), d\left(x_{n}, x_{n+1}\right), d\left(x_{n-1}, x_{n}\right), d\left(x_{n}, x_{n}\right), d\left(x_{n+1}, x_{n-1}\right)\right\}\right),\right. \\
&\left.\varphi\left(r \max \left\{d\left(x_{n}, x_{n-1}\right), d\left(x_{n}, x_{n+1}\right), d\left(x_{n-1}, x_{n}\right), d\left(x_{n}, x_{n}\right), d\left(x_{n+1}, x_{n-1}\right)\right\}\right)\right) \\
& \leq F\left(\psi\left(r \max \left\{d\left(x_{n}, x_{n-1}\right), d\left(x_{n}, x_{n+1}\right), d\left(x_{n+1}, x_{n-1}\right)\right\}\right),\right. \\
&\left.\varphi\left(r \max \left\{d\left(x_{n}, x_{n-1}\right), d\left(x_{n}, x_{n+1}\right), d\left(x_{n+1}, x_{n-1}\right)\right\}\right)\right) \\
& \leq F\left(\psi\left(r \max \left\{d\left(x_{n}, x_{n-1}\right), d\left(x_{n+1}, x_{n-1}\right)\right\}\right), \varphi\left(r \max \left\{d\left(x_{n}, x_{n-1}\right), d\left(x_{n+1}, x_{n-1}\right)\right\}\right)\right)
\end{aligned}
$$


$\leq \psi\left(r \max \left\{d\left(x_{n}, x_{n-1}\right), d\left(x_{n+1}, x_{n-1}\right)\right\}\right.$

Case (i) If $d\left(x_{n+1}, x_{n}\right) \leq r d\left(x_{n}, x_{n-1}\right)$ then we get, $d\left(x_{n+1}, x_{n}\right) \leq r^{n} d\left(x_{1}, x_{0}\right)$ for $n>m$

$d\left(x_{n}, x_{m}\right) \leq d\left(x_{n}, x_{n-1}\right)+d\left(x_{n-1}, x_{n-2}\right)+\cdots+d\left(x_{m+1}, x_{m}\right)$

$\leq\left[r^{n-1}+r^{n-2}+\cdots+r^{m}\right] d\left(x_{1}, x_{0}\right)$

$\leq \frac{r^{m}}{(1-r)} d\left(x_{1}, x_{0}\right)$

We get $\left\|d\left(x_{n}, x_{m}\right)\right\| \leq K \frac{r^{m}}{(\mathbf{1}-\boldsymbol{r})} \| d\left(x_{1}, x_{0}\right) . d\left(x_{n}, x_{m}\right) \rightarrow 0$ as $n, m \rightarrow \infty$. Hence $\left\{x_{n}\right\}$ is a Cauchy sequence. By the completeness of $X$, there is $p \in X$. such that $x_{n} \rightarrow p$ as $n \rightarrow \infty$

$\psi(d(T p, P)) \leq \psi\left(H\left(T x_{n}, T p\right)+d\left(T x_{n}, p\right)\right)$

$\leq F\left(\psi\left(r \max \left\{d\left(x_{n}, p\right), d\left(x_{n}, T x_{n}\right), d(p, T p), d\left(x_{n}, T p\right), d\left(T x_{n}, p\right)\right\}+d\left(x_{n+1}, p\right)\right)\right.$, $\left.\varphi\left(r \max \left\{d\left(x_{n}, p\right), d\left(x_{n}, T x_{n}\right), d(p, T p), d\left(x_{n}, T p\right), d\left(T x_{n}, p\right)\right\}+d\left(x_{n+1}, p\right)\right)\right)$

$\leq F\left(\psi\left(r \max \left\{d\left(x_{n}, p\right), d\left(x_{n}, x_{n+1}\right), d(p, T p), d\left(x_{n}, T p\right), d\left(x_{n+1}, p\right)\right\}+d\left(x_{n+1}, p\right)\right)\right.$, $\left.\varphi\left(r \max \left\{d\left(x_{n}, p\right), d\left(x_{n}, x_{n+1}\right), d(p, T p), d\left(x_{n}, T p\right), d\left(x_{n+1}, p\right)\right\}+d\left(x_{n+1}, p\right)\right)\right)$

$\leq F(\psi(r d(p, T p)), \varphi(r d(p, T p)))$

$d(T p, P)=0$. Hence $P \in T p$

Case (ii) $d\left(x_{n+1}, x_{n}\right) \leq r d\left(x_{n+1}, x_{n-1}\right)$ then we get

$d\left(x_{n+1}, x_{n}\right) \leq r\left[d\left(x_{n+1}, x_{n}\right)+d\left(x_{n}, x_{n-1}\right)\right]$

$\leq \frac{r}{1-r}\left[d\left(x_{n}, x_{n-1}\right)\right]$

$\leq h\left[d\left(x_{n}, x_{n-1}\right)\right] \quad$ where $h=\frac{r}{1-r}<1$

For $n>m$

$$
\begin{aligned}
d\left(x_{n}, x_{m}\right) & \leq d\left(x_{n}, x_{n-1}\right)+d\left(x_{n-1}, x_{n-2}\right)+\cdots+d\left(x_{m+1}, x_{m}\right) \\
& \leq\left[h^{n-1}+h^{n-2}+\cdots+h^{m}\right] d\left(x_{1}, x_{0}\right) \\
& \leq \frac{h^{m}}{(1-h)} d\left(x_{1}, x_{0}\right)
\end{aligned}
$$

We get $\left\|d\left(x_{n}, x_{m}\right)\right\| \leq K \frac{h^{m}}{(1-h)} \| d\left(x_{1}, x_{0}\right) . d\left(x_{n}, x_{m}\right) \rightarrow 0$ as $n, m \rightarrow \infty$. Hence $\left\{x_{n}\right\}$ is a Cauchy sequence. By the completeness of $X$, there is $p \in X$. such that $x_{n} \rightarrow p$ as $n \rightarrow \infty$

$\psi(d(T p, P)) \leq \psi\left(H\left(T x_{n}, T p\right)+d\left(T x_{n}, p\right)\right)$

$\leq F\left(\psi\left(r \max \left\{d\left(x_{n}, p\right), d\left(x_{n}, T x_{n}\right), d(p, T p), d\left(x_{n}, T p\right), d\left(T x_{n}, p\right)\right\}+d\left(x_{n+1}, p\right)\right)\right.$, $\left.\varphi\left(r \max \left\{d\left(x_{n}, p\right), d\left(x_{n}, T x_{n}\right), d(p, T p), d\left(x_{n}, T p\right), d\left(T x_{n}, p\right)\right\}+d\left(x_{n+1}, p\right)\right)\right)$

$\leq F\left(\psi\left(r \max \left\{d\left(x_{n}, p\right), d\left(x_{n}, x_{n+1}\right), d(p, T p), d\left(x_{n}, T p\right), d\left(x_{n+1}, p\right)\right\}+d\left(x_{n+1}, p\right)\right)\right.$,

$\left.\varphi\left(r \max \left\{d\left(x_{n}, p\right), d\left(x_{n}, x_{n+1}\right), d(p, T p), d\left(x_{n}, T p\right), d\left(x_{n+1}, p\right)\right\}+d\left(x_{n+1}, p\right)\right)\right)$

$\leq F(\psi(r d(p, T p)), \varphi(r d(p, T p)))$

$d(T p, P)=0$. Hence $P \in T p$

$$
\begin{aligned}
\psi(d(p, q))= & \psi(H(T p, T q)) \\
\leq & F(\psi(r \max \{d(x, y), d(p, T p), d(q, T q), d(p, T q), d(T p, q)\}), \\
& \varphi(r \max \{d(x, y), d(p, T p), d(q, T q), d(p, T q), d(T p, q)\})) \\
\leq & F(\psi(r \max \{d(p, q), d(p, p), d(q, q), d(p, q), d(p, q)\}), \\
& \varphi(r \max \{d(p, q), d(p, p), d(q, q), d(p, q), d(p, q)\})) \\
\leq & F(\psi(r[d(p, q)]), \varphi(r[d(p, q)])) \\
\leq & \psi(r[d(p, q)])
\end{aligned}
$$

This is contradiction and hence $T$ has a unique fixed point in $X$

Corollary 2.3: Let $(X, d)$ be a complete cone metric space and $P$ a normal cone with normal constant $K$. Suppose the mapping $T: X \rightarrow C B(X)$ be multivalued map satisfy the condition

$$
\psi(H(T x, T y)) \leq F(\psi(r \max \{d(x, y), d(x, T x), d(y, T y)\}), \varphi(r \max \{d(x, y), d(x, T x), d(y, T y)\}))
$$

For all $x, y \in X$ and $r \in[0,1).) . \psi$ and $\varphi$ are altering distance and ultra altering distance functions respectively, $F \in \mathcal{C}$ such that $\psi(t+s) \leq \psi(t)+\psi(s)$. Then $T$ has a fixed point in $X$

Proof: The proof of the corollary immediately follows since

$$
\max \{d(x, y), d(x, T x), d(y, T y)\} \leq \max \{d(x, y), d(x, T x), d(y, T y), d(x, T y), d(T x, y)\}
$$

\section{REFERENCES}


[1]. R. Krishnakumar, M. Marudai “Fixed Point Theorems of Multivalued Mappings in Cone Metric Spaces” Int. J. Contemp. Math. Sciences, Vol. 5, 2010, no. 31, $1533-1540$

[2]. Huang Gaung,Zhang Xian,"Cone metric spaces and fixed point theorems of contractive mappings" J.math.Anal. Appl.332(2007)1468-1476.

[3]. H.Mohebi,Topical functions and their properties in a class of ordered Banach spaces, in continuous Optimization,Current Trends and Modern Applications, PartII, Springer, 2005.pp.343-361.

[4]. H.Mohebi,H.Sadeghi,A.M.Rubinov,Best approximation in a class of normed spaces with star-shaped cone, Numer. Funct.Anal.Optim.27(34)(2006)411- 436.

[5]. Sh.Rezapour,R.Hamlbarani,"Some notes on the Paper-Cone metric Spaces and fixed point theorems of contractive mappings,"J.Math.Anal.Appl.345 (2008)719-724.

[6] Seong Hoon Cho,Mi Sun Kim,"Fixed point theorems for general contractive multivalued mappings,"-J.Appl.Math.Informatics Vol.27 (2009)343- 350.

[7] R.Krishnakumar, K. Dinesh, Fixed point of cyclic contraction functions in banach spaces, International Journal Of Innovative Research In Science ,Engineering And Technology, Vol. 5, Issue 7, July 2016, Pg-13316 -13319

[8] A. H. Ansari, "Note on $\phi-\psi$-contractive type mappings and related fixed point,"The 2 nd Regional Conference on MathematicsAndApplications, PNU, September(2014), 377-380.

[9] Arslan Hojat Ansari, Sumit Chandok, Nawab Hussin and Ljiljana Paunovic, "Fixedpoints of ( $\psi$, $\varphi)$-weak contractions in regular cone metric spaces via new function”, J. Adv. Math. Stud. Vol. 9(2016), No. 1, 72-82.

[10] R.Krishnakumar, K. Dinesh, "Existence of fixed points of fuzzy functions in complete metric spaces", Proceedings of the International conference on applied mathematical models (Pg:240-242), ICAMM 2014 PSG College of Technology, Coimbatore, India

[11] R.Krishnakumar, K. Dinesh, "Fixed Point Theorems of Fuzzy Function in Complete Metric Spaces", International Journal Of Innovative Research In Science, Engineering And Technology, Vol. 6, Issue 7, July 2017, Pg-13557-13562

[12] R. Krishnakumar, K. Dinesh, "Fuzzy Mapping and their Fixed Point Theorems in Complete Metric Spaces"International Journal of Scientific Research in Science, Engineering and Technology, Volume 3 , Issue 8, November-December-2017, Pg: 241-249

[13] Arslan Hojat Ansari, R. Krishnakumar, K. Dinesh,_and D. Dhamodharan, "Fixed Point of Cyclic Contraction Mappings in Banach Spaces Via C-Class Function" Int. J. Math. And Appl., 6(1-E)(2018), 1085-1092

[14] R. Krishnakumar, K. Dinesh, D. Dhamodharan, "Some Fixed Point Theorems in $\varphi-\psi$ weak contraction on Fuzzy Metric Space" Int. J. Sci. Res. in Mathematical and Statistical Sciences Vol. 5(3), Jun 2018

\section{AUTHORS PROFILE}

Dr. R. Krishnakumar is the Associate Professor and Head of the PG \& Research Department of Mathematics, Urumu Dhanalakshmi College. He has above 22 years experience in teaching. His field of research is Fixed point theory.

K. Dinesh is pursuing his Ph.D in PG \& Research Department of Mathematics, Urumu Dhanalakshmi College, Trichy. His field of research is Fixed point theory.

Arslan Hojat Ansari is the assistant professor, Karaj Branch, Islamic Azad University, Karaj, Iran. 